\title{
Diagnóstico Pré-Natal de Duplo Arco Aórtico
}

Antenatal Diagnosis of Double Aortic Arch

Natália Noronha, ${ }^{10}$ Angie Hobbs, ${ }^{10}$ Patricia Caldas ${ }^{1}$

Bristol Royal Hospital for Children, ${ }^{1}$ Bristol - Reino Unido da Grã-Bretanha

\section{Introdução}

As anomalias congênitas do arco aórtico afetam 1-2\% da população e compreendem uma grande variedade de anormalidades na posição e / ou padrão de ramificação do arco aórtico. ${ }^{1} \mathrm{O}$ duplo arco aórtico (DAA) representa 1-2\% de todas as anomalias do arco aórtico e é caracterizado pela persistência dos arcos aórticos embrionários esquerdo e direito. ${ }^{2}$ A anomalia pode ser encontrada isoladamente ou, com menor frequência, em associação com outras anormalidades cardiovasculares ou cromossômicas. O diagnóstico pré-natal de DAA pode ser desafiador, pois sua diferenciação de outras anormalidades do arco, como arco aórtico direito com canal arterial esquerdo ou com artéria subclávia esquerda aberrante, nem sempre é clara. Os autores descrevem um caso de diagnóstico pré-natal de DAA.

\section{Relato de Caso}

Uma primigesta de 26 anos foi encaminhada para uma revisão de cardiologia fetal com $23+3$ semanas de gestação devido a uma imagem anormal dos três vasos e traqueia (3VT) em um exame de ultrassonografia morfológica de rotina com 20 semanas de gravidez. Ela não estava tomando medicamentos e não havia histórico pessoal ou familiar relevante.

O ecocardiograma fetal mostrou projeção de quatro câmaras e vias de saída normais. Na projeção de 3VT, entretanto, o canal arterial foi visto do lado esquerdo da traqueia e o arco aórtico do lado direito, confirmando a presença de um arco aórtico direito com canal arterial esquerdo (Figura 1). Com uma imagem mais próxima, notou-se uma estrutura menor do lado esquerdo da traqueia, circundando a mesma completamente. Para confirmação do diagnóstico de DAA, as artérias subclávias foram localizadas e cada uma foi vista originando-se do respectivo arco aórtico (Figura 2). Não havia sinais de obstrução em nenhum dos

\section{Palavras-chave}

Cardiopatias Congênitas/diagnóstico; Cardiopatias Congênitas/cirurgia; Aorta Torácica/diagnosis; Heart Defects Congenital/surgery; Aberrações Cromossômicas; Ultrassonografia/métodos; Ecocardiografia/métodos; Paralisia das Cordas Vocais; Broncomalácia/congênito.

\section{Correspondência: Natália Noronha •}

Fetal Cardiology Service, Bristol Royal Hospital for Children, Bristol, United Kingdom - Upper Maudlin St Bristol BS2 8BJ - Reino Unido da Grã-Bretanha E-mail: natalianoronhaf@gmail.com

Artigo recebido em 11/05/2019, revisado em 01/09/2020, aceito em 26/11/2019

DOI: https://doi.org/10.36660/abc.20190310 arcos aórticos. Nenhuma outra anormalidade cardíaca ou extracardíaca foi encontrada. Dada a possível associação de DAA com anormalidades cromossômicas, particularmente a microdeleção 22q11.2, o casal foi aconselhado a se submeter a testes invasivos, os quais eles recusaram.

Após o parto, um exame de imagem pós-natal confirmou os achados pré-natais (Figura 3). Aos 2 meses de idade, os pais notaram estridor leve intermitente. $\mathrm{O}$ paciente foi encaminhado para uma tomografia computadorizada cardíaca, que confirmou o diagnóstico de duplo arco aórtico com atresia do arco distal esquerdo (Figura 4). O paciente foi submetido à separação cirúrgica do arco esquerdo aos 3 meses de idade, complicada por paralisia de prega vocal esquerda.

O paciente tem atualmente 5 meses de idade, apresenta estridor intermitente por broncomalácia e paralisia de prega vocal esquerda e é alimentado por sonda nasogástrica.

\section{Discussão}

O DAA é o substrato mais frequente para um anel vascular e pode resultar em sintomas respiratórios e / ou digestivos desde tenra idade. Na maioria dos casos, um dos arcos é dominante, mais frequentemente o direito (em pelo menos $75 \%$ dos casos). ${ }^{2}$ Pode haver um segmento de atresia em um ou vários locais em qualquer um dos dois arcos, geralmente o esquerdo, ${ }^{1,3}$ como aconteceu em nosso caso. O DAA resulta em sintomas respiratórios como estridor, episódios de asfixia e infecções recorrentes do trato respiratório em $91 \%$ dos pacientes. Os sintomas gastrointestinais, por outro lado, ocorrem em $40 \%$ dos casos e incluem vômitos, intolerância alimentar em lactentes e disfagia em crianças maiores e adultos. ${ }^{2,4}$

O diagnóstico de DAA pode ser feito através da projeção de 3VT descrita por Yagel et al., ${ }^{5}$ Nesta projeção, o arco aórtico normal (esquerdo) é observado à esquerda da linha média e da traqueia. O canal arterial é visto lateralmente no seu lado esquerdo. O arco aórtico e o canal arterial convergem então em uma estrutura em forma de $\mathrm{V}$ que continua como a aorta descendente. O terceiro vaso que compreende a projeção de 3VT é a veia cava superior, que é vista à direita da linha média. Em um arco aórtico esquerdo normal, nenhuma estrutura vascular importante é vista cruzando a traqueia posteriormente. Por outro lado, no DAA, a projeção de 3VT representa um arco aórtico esquerdo e direito, formando um anel vascular que circunda completamente a traqueia. Este anel, junto com o canal arterial, forma a figura de um " 6 " ou um "9" (também descrito como formato de tridente) em vez da estrutura clássica em forma de $V$ descrita acima. A presença de fluxo anterógrado em ambos os arcos e no canal arterial pode ser confirmada pelo mapeamento de fluxo em cores. Este último também deve ser utilizado para confirmar ou excluir obstrução ao fluxo em qualquer um dos arcos. 


\section{Relato de Caso}
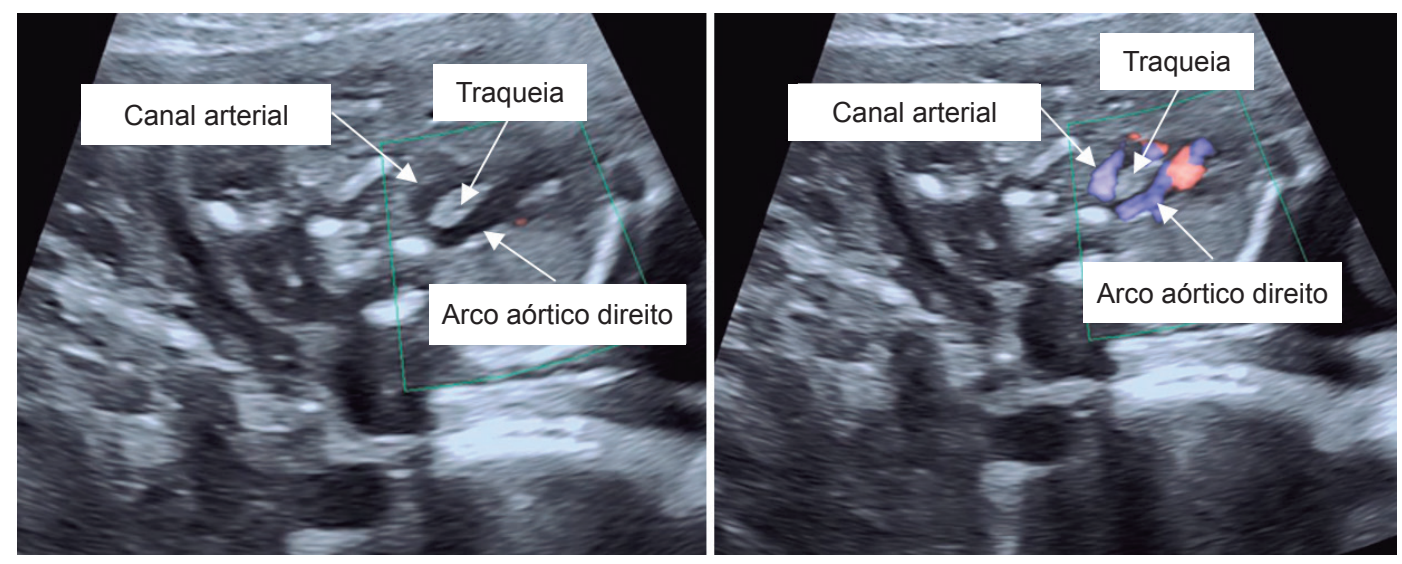

Figura 1 - Ecocardiograma fetal (projeção dos três vasos e traqueia) mostrando um canal arterial esquerdo e direito circundando completamente a traqueia sem (painel esquerdo) e com (painel direito) mapeamento de fluxo em cores.

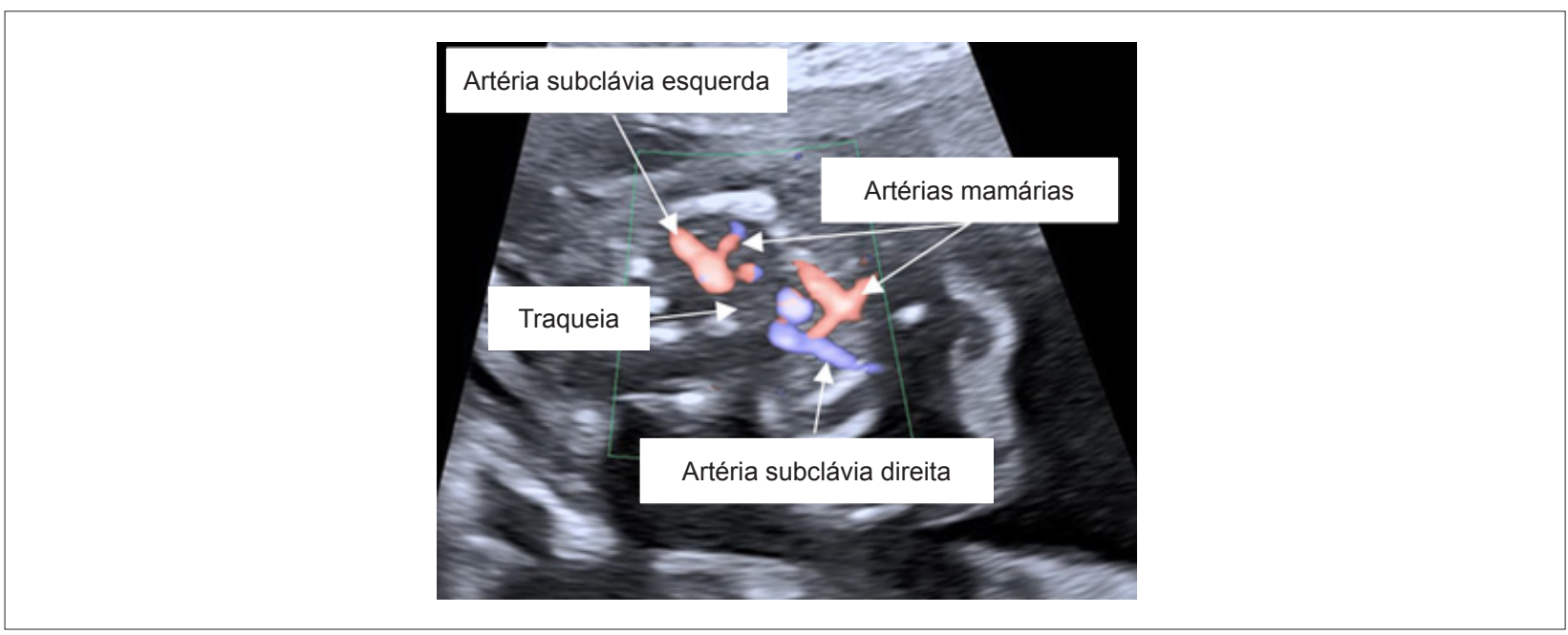

Figura 2 - Ecocardiograma fetal (imagem axial de fluxo em cores) mostrando as artérias subclávias direita e esquerda originando-se dos arcos aórticos esquerdo e direito, respectivamente.

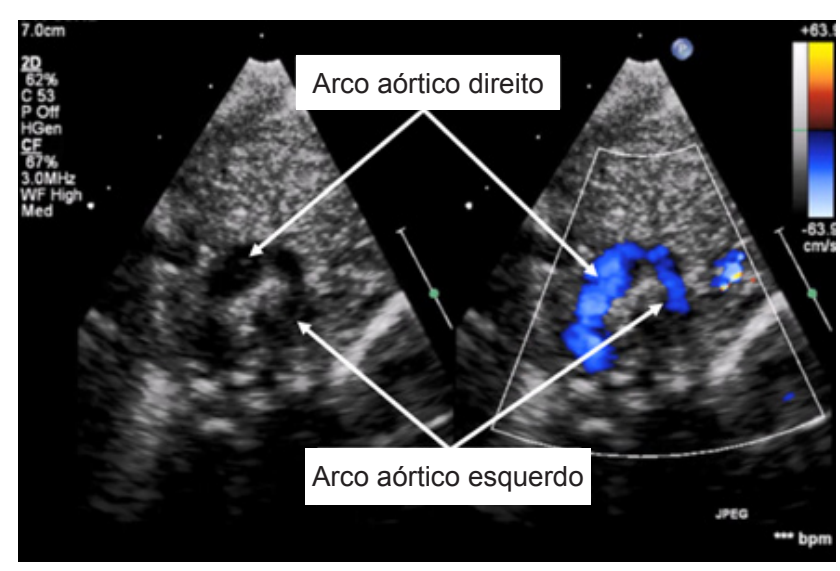

Figura 3 - Ecocardiograma transtorácico - projeção paraesternal alta (2D e mapeamento de fluxo em cores) mostrando o arco aórtico direito dominante e o menor esquerdo. 


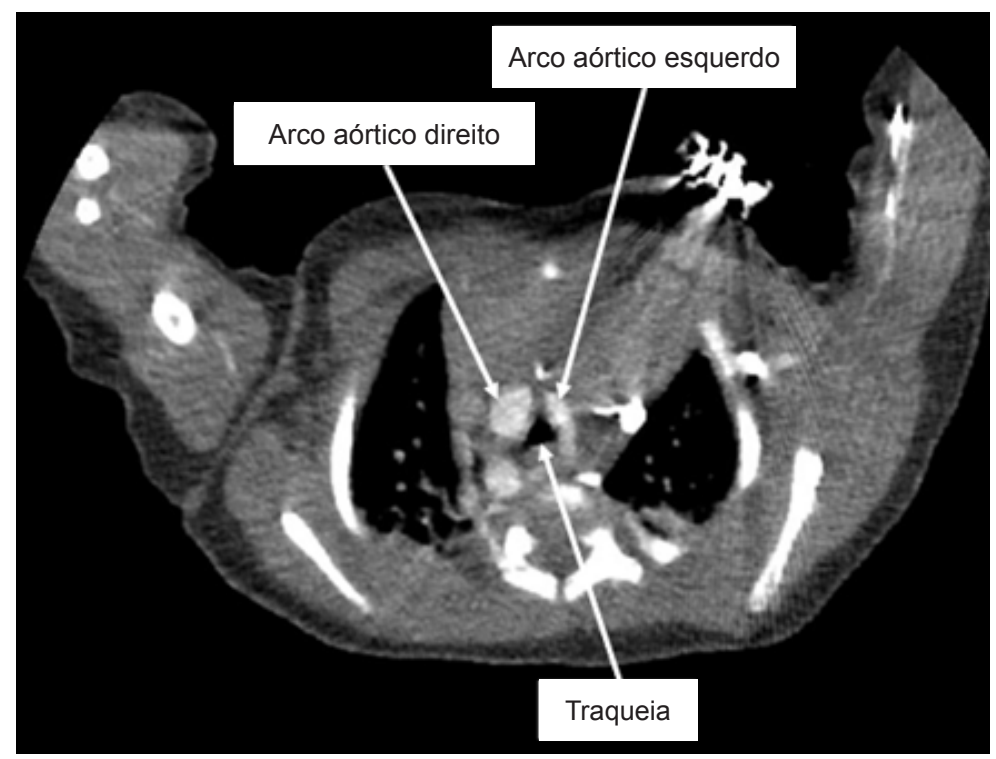

Figura 4 - Tomografia computadorizada - imagem axial mostrando o arco aórtico direito dominante e o menor esquerdo.

Pode ser difícil diferenciar o DAA de outras anormalidades do arco aórtico, como arco aórtico direito com canal arterial esquerdo na projeção de 3VT. Nessa situação, a identificação da origem das artérias subclávias pode auxiliar no diagnóstico diferencial. Se cada uma das artérias subclávias for vista surgindo dos arcos aórticos esquerdo e direito (para os lados esquerdo e direito da traqueia, respectivamente), o diagnóstico de DAA pode ser estabelecido.

Foi relatado que o reparo cirúrgico precoce do DAA elimina os sintomas em mais de 70\% dos casos, embora a limitação do fluxo de ar possa persistir devido à estenose traqueal residual. ${ }^{2}$ Em uma revisão de 183 pacientes com anéis vasculares submetidos à correção cirúrgica, ${ }^{6} 2$ pacientes necessitaram de traqueostomia por grave compressão distal da traqueia e um paciente apresentou paralisia de prega vocal esquerda verdadeira, como ocorreu em nosso caso.

Embora desafiador, o diagnóstico pré-natal de DAA permite uma caracterização oportuna do anel vascular e facilita o planejamento da intervenção cirúrgica antes ou logo após o desenvolvimento dos sintomas. Embora os sintomas possam não ser resolvidos imediatamente, uma separação precoce do DAA é crucial para prevenir sequelas de longo prazo de compressão traqueobrônquica e dificuldades de alimentação. ${ }^{2}$

\section{Contribuição dos Autores}

Obtenção de dados: Hobbs A; Redação do manuscrito: Noronha N; Revisão crítica do manuscrito quanto ao conteúdo intelectual importante: Caldas P.

\section{Potencial Conflito de Interesses}

Declaro não haver conflito de interesses pertinentes.

\section{Fontes de Financiamento}

O presente estudo não teve fontes de financiamento externas.

\section{Vinculação Acadêmica}

Não há vinculação deste estudo a programas de pósgraduação.

\section{Aprovação Ética e Consentimento Informado}

Este artigo não contém estudos com humanos ou animais realizados por nenhum dos autores. 


\section{Relato de Caso}

\section{Referências}

1. Trobo D, Bravo C, Alvarez T, Pérez R, Gámez F, León-Luis J. Prenatal Sonographic Features of a Double Aortic Arch: Literature Review and Perinatal Management. J Ultrasound Med. 2015;34(11):1921-7.

2. Gou Z, He Y, Zhang Y, Gu X. Prenatal diagnosis of fetal double aortic arch: report of a case. Int J Clin Exp Pathol. 2015;8(10):13565-7.

3. Schlesinger AE, Krishnamurthy R, Sena LM et al. Incomplete double aortic arch with atresia of the distal left arch: distinctive imaging appearance. AJR Am J Roentgenol. 2005;184(5):1634-9.
4. Krishnasarma R, Green Golan Mackintosh L, Bynum. ALTE and Feeding Intolerance as a Presentation of Double Aortic Arch. Case Rep Pediatr. 2016; 2016(5):1-3.

5. Yagel S, Arbel R, Anteby EY, Raveh D, Achiron R. The three vessels and trachea view (3VT) in fetal cardiac scanning. Ultrasound Obstet Gynecol. 2002 Oct; $20(4): 340-5$

6. Ruzmetov M, Vijay P, Rodefeld MD, Turrentine MW, Brown JW. Follow-up of surgical correction of aortic arch anomalies causing tracheoesophageal compression: a 38-year single institution experience. J Pediatr Surg. 2009;44(7):1328-32. 